\title{
Design and Evaluation of a Low-Power Sensor Device for Induced Rockfall Experiments
}

\author{
Andrin Caviezel ${ }^{1}$, Michael Schaffner ${ }^{2}$, Lukas Cavigelli ${ }^{2}$, Pascal Niklaus ${ }^{2}$, Yves Bühler ${ }^{1}$, \\ Perry Bartelt ${ }^{1}$, Michele Magno ${ }^{2}$, and Luca Benini ${ }^{2}$ \\ ${ }^{1}$ WSL Institute for Snow and Avalanche Research SLF, Davos, Switzerland, Email: surname@slf .ch \\ ${ }^{2}$ ETH Zurich, Integrated Systems Lab IIS, Zurich, Switzerland, Email: surname@i is.ee.ethz.ch
}

\begin{abstract}
Rockfalls have over the last decades become a serious and frequent hazard, especially due to larger variations in precipitation and temperatures, destabilizing rocky slopes in mountainous regions. Hence, civil engineers are applying the latest simulation tools to perform risk assessments and plan mitigation strategies. These tools are based on various models with many parameters that should be calibrated and evaluated with real-world in-field measurement data.

In this work, we present a rugged low-power multi-sensor node termed StoneNode, that has been designed to acquire and $\log$ accurate inertial sensor measurements during induced infield experiments with falling rocks. The node hosts low-power MEMS sensors with high dynamic ranges sampled up to $1 \mathrm{kHz}$, and provides a long battery life-time of up to $56 \mathrm{~h}$, enabling long-lasting field studies with a duration of several working days. Exhaustive in-field experiments have been carried out with several differently shaped rocks on typical terrain in the Swiss alpine region. The experiments comprise more than 100 induced tests with several heavy impacts of $>400 \mathrm{~g}$. This paper gives a detailed summary of these results, including unprecedented insitu data of rock fall trajectories and post-experimental validation where we compare simulated rockfall deposition distributions and motion traces with in-field measurements after calibration of the simulation module.

Our results and experience gained in-field confirm that the StoneNode is a reliable, easy-to-use device, which greatly facilitates the data acquisition process. Further, the results obtained with the calibrated simulation tool shows good quantitative and qualitative congruence with the experiments, further reaffirming our methodological approach.
\end{abstract}

Keywords-Rockfall, low-power, sensor node architecture, data logger, MEMS sensors.

\section{INTRODUCTION}

Rockfall is one of most dangerous natural hazards especially in mountainous regions such as the Alpine region in Europe [1-3]. It threatens transiting road traffic, railways, buildings and human infrastructure. Almost every year many railway transit lines through the Alps are interrupted for several weeks due to rockfall damage, incurring delays and high costs for transport companies and threatening the lives of people. Meshes draped over the slope faces and barrier fences are often used to protect streets and buildings from rockfalls [4], [5]. However, in many case these protections are not enough, hence tunnel-like protections have to be constructed, especially for highways and railway tracks. A more expensive
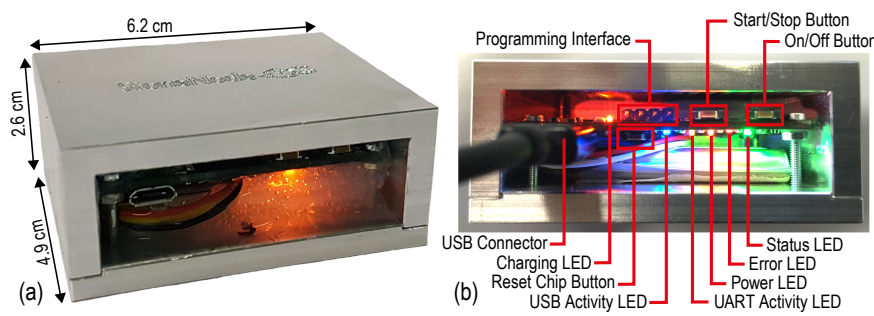

Fig. 1. (a) StoneNode molded into case with resin, (b) close-up of interfaces.

protection consists in slope modifications such as berms, rock sheds, ditches or fills, which reduces the threats. The main drawback of these protections is the high cost of planning, installation and construction, which is usually financed by the local governments. This leads to a delicate trade-off between the immediate financial burden of the protection measures and the safety risks involved.

Simulation and estimation software solutions based on mechanical models have been investigated and developed recently [6-14]. Today, state-of-the-art solutions are able to perform accurate rockfall trajectories simulations, and they are crucial tools for civil engineers to improve the risks assessments and develop mitigation strategies [6], [7], [15]. These sophisticated simulation tools are based on high quality mass movement and dynamics models, coupled with precise terrain information. These models have many parameters, and to achieve reliable simulations, these parameters need to be calibrated and validated using in-field experiments. Inaccurate modeling caused by insufficiently calibrated models or caused by inexperienced users may lead to the application of large safety margins, which in turn increases cost and construction complexity of the planned protection measures, or even more fatal, to an underestimation of the risk.

As mentioned, to achieve accurate simulation models, the crucial task is to obtain accurate measurements of the rock trajectories [16]. Today, there is no data set consisting of highquality measurements available, and hence the only solution is to acquire such data by logging them in in-field experiments. Many research institutes, such as the WSL Institute for Snow and Avalanche Research (SLF) in Davos, Switzerland, are currently attempting to acquire such data in Alpine terrains to improve their simulation models. To this end, the most relevant parameters are the rock materials, the terrain shape and

This document is the accepted manuscript version of the following article:

Cavieze1, A., Schaffner, M., Cavigel1i, L., Niklaus, P., Bühler, Y., Barte1t, P., ... Benini, L. (2018). Design and evaluation of a low-power sensor device for induced rockfall experiments. IEEE Transactions on Instrumentation and Measurement, 67(4), 767-779. 
ground properties such as elasticity, and the trajectories and incurred accelerations and angular velocities of typical rocks in motion. Terrain models are usually acquired with aerial photogrammetry using Unmanned Aerial Systems (UASs) and material parameters of the ground can be measured with specialized instruments. Trajectory samples and forces are typically acquired with induced rockfall experiments, using either video capturing systems or in-situ sensors [17].

In the last decade, miniature and low-power microelectromechanical system (MEMS) inertial sensors have become popular to measure motion attributes such as velocities and accelerations. An interesting category of MEMS are the inertial measurement units (IMUs) which have been used to capture data for a variety of applications [18]. Recent IMU modules provide precise measurements with high sampling rates and the fusion of inertial sensor data with data stemming from other sensor types (e.g., pressure) has been demonstrated to increase accuracy in many fields [19-21]. To deploy such sensors during in-field experiments, battery-powered sensor nodes have to be designed in a robust and reliable manner and with low energy consumption to enable several experiment repetitions with the same hardware.

Contributions: In this paper, we present an updated version of the StoneNode that has initially been presented in [22]. The StoneNode is a low-power sensor node containing embedded MEMS sensors to log accurate measurements of moving rocks in induced field experiments. The updated version of the node shares the same main architecture and components of the previous one, but it includes a pressure sensor for measuring altitude differences, which is useful for assessing the height of the jumps in the rockfall trajectory. Furthermore, the updated firmware enables higher accelerometer and gyroscope sampling rates of up to $1 \mathrm{kHz}$ instead of 400 and $500 \mathrm{~Hz}$, respectively. A major contribution of this work are the extensive measurements (accelerations and angular velocities) acquired with the StoneNode by instrumenting several differently shaped rocks on a test site in the Swiss alps. Moreover we present a comprehensive description of the experimental setup and the rocks that have been used, including initial results and comparisons of ongoing calibration and validation efforts of the RAMMS::ROCKFALL simulation software [6], [7].

\section{RELATED WORK}

In this section, we present existing work on current software tools and simulation models for rockfall simulation, and provide an overview of typical experimental measurement setups and data collection methods, including in situ sensor nodes.

\section{A. Rockfall Simulation Software}

Accurate prediction of mass movement, run-out distances, flow velocities and height, and impact pressures in natural three-dimensional terrain is the driving motivation behind the development of improved mass movement dynamics models. There are several simulation programs and rockfall models available, such as the Colorado Rockfall Simulation Program [14], Rockyfor3D [23], RocFall [24], and RAMMS::ROCKFALL [6], [7]. The last one is built around the well-established Rapid Mass Movement Simulation (RAMMS) for avalanches and debris flow and provides the current stateof-the-art 3D rigid body and slippage model, capable of simulating various complex rock shapes and modeling the impact of terrain scaring to provide highly accurate estimates of trajectory probability and potential range. The modules for avalanches and debris flow have already been extensively verified by experiments on real-scale test sites in Vallée de la Sionne and Illgraben, Switzerland, and data from numerous data sets of historic avalanche and debris flow events were used for development and calibration. While the released edition of RAMMS::ROCKFALL has been extensively calibrated via back calculation of case studies, further developments and extensions need more data for further improvement. With the development of the StoneNode we provide an easy-to-use device for this task.

\section{B. Data Collection Methods for Model Verification}

Various approaches to measure rockfall trajectories have been presented in [25], [26], with concepts involving in situ measurement devices, local positioning systems, and videobased tracking with multiple high-speed cameras and fixed visual location marking around the test slope of about $200 \mathrm{~m}$ length and $50 \mathrm{~m}$ width. Another series of experiments has been conducted by [27], where visual tracking of the falling rock has been used on a small test site. They used a single high-resolution, high frame rate camera and many reference point markings on the slope and on the rock for tracking. The test site for these experiment was limited to a few $10 \mathrm{~s}$ of meters and the rocks were released from a height of $6 \mathrm{~m}$ above the ground. In [28], rockfall experiments with tracking using multiple cameras have been undertaken to verify some rockfall models on site with a length of about $500 \mathrm{~m}$ and an elevation difference of about $250 \mathrm{~m}$.

The aforementioned approaches all require complex on-site setups to fix visual reference points. Because of the limited resolution of even high-end machine vision cameras over long distances, a single camera strongly limits the size of the test site and a multi-camera setup requires complex data extraction. In neither setup a 3D trajectory reconstruction is performed, in fact [26] use position measurements projected onto a horizontal plane, and [28] view the 2D jump trajectory from the side.

In-situ measurement devices are easier to set up and do not exhibit the mentioned limitations. Early experiments by the SLF with such devices have been published in [25], [26]. These devices make use of accelerometers, gyroscopes and local positioning systems (LPS), and the newest sensor from [26] can measure accelerations up to $\pm 500 \mathrm{~g}$ and angular velocities up to $500 \%$ s, both with 8 bit resolution at around $900 \mathrm{~Hz}$. The LPS provides position updates at around $15 \mathrm{~Hz}$ and requires several external antennas to be distributed over the testing site. All sensors developed in [25], [26] use dated technology which is not densely integrated, large and does not always provide sufficient resolution and sampling rate. Also, the sensor nodes are large in size, consist of several connected PCBs and have limited storage and battery capacity, making them inconvenient to handle in the field. A miniaturized and low-range sensor node is presented in [29] labelled SmartStone based on a Bosch Sensortex BMX055 IMU. Being designed for 
rather slow motions it fulfills its metering purpose for pebbles but is not suited for fast motions and heavy impacts.

Almost all commercial sensor nodes which are available today (sometimes also referred to as motes [30]) are not suitable for the task at hand due to the harsh environment and strict specifications of the application (high dynamic sensor ranges, industrial grade). The often used 'extension shields' have to be mechanically connected to the mote base boards, which leads to larger size and unreliable designs. The single commercially available sensor node covering the desired metering range is the TSR 6DXP by Diversified Technical Systems. Its high price of $>10 \mathrm{k}$ USD per device and nonexistent upgrade possibilities due to the proprietary design makes this device rather inappropriate for use as a possibly expendable device in rockfall experiments. Table I gives a qualitative overview of existing methods and devices to identify rock movements with respect to their measured degrees of freedom (DOF), their respective ranges for the measured parameters, the data access (where a simple plug an play retrieval is favoured), its upgrade capabilities and its costs. Clearly, devices employing new sensor fusion technologies feature more advantages. Thus, developing a customized node allows to use the lowest amount of components without mechanical parts and ensures that the specifications are met. The StoneNode represents a continuation of the work by SLF, and specifically focuses on the aforementioned issues by reducing the power consumption in order to increase operating lifetime, by improving on the form factor and ruggedness, and by designing interfaces which allow convenient data retrieval. The developed sensor node will serve as a basis for a new generation of rockfall sensors which are easier to handle and which employ modern technology.

\section{III.STONENOde System OVERVIEW}

This paper presents StoneNode, a low-power, robust and reliable multi-sensor node with a fast accessible interface. The StoneNode comes in two versions: the base version v1.0 that has been initially presented in [22] and an updated version v1.1. As indicated in the top-level block diagram shown in Figure 2, both StoneNode versions share the same main architectural features. The main differences of v1.1 with respect to v1.0 are two additional components: a barometer for direct measurement of altitude differences, and a buzzer for more convenient retrieval in the field experiments in case of rock disintegration. Further, the firmware has been improved in order to support higher accelerometer and gyroscope sampling

TABle I. Qualitative Comparison of Available Methods and DEVICES FOR MEASURING ROCKFALL MOTION.

\begin{tabular}{lccccc}
\hline Method/Device & DOF & Range & Access & Upgrade & Costs \\
\hline High Speed Video & - & + & --- & - & -- \\
LPS & + & - & -- & - & - \\
Radar & + & + & --- & -- & --- \\
SmartStone & ++ & --- & +++ & --- & +++ \\
Commercial Motes & +++ & --- & +++ & +++ & ++ \\
DTS TSR 6DXP & ++ & +++ & +++ & --- & -- \\
StoneNode v1.0 & ++ & ++ & +++ & +++ & +++ \\
StoneNode v1.1 & +++ & ++ & +++ & +++ & +++ \\
\hline
\end{tabular}

rates of $1 \mathrm{kHz}$, and the aluminum case has been extended with a lid for improved protection during field tests.

The node works with a $1100 \mathrm{mAh}$ LiPo battery for $20-56 \mathrm{~h}$ (depending on the employed sensor configuration and sampling rates) and stores all the information on an on-board flash memory. An USB port allows to recharge the battery, provides access to the recorded data, and debugging capabilities. Highspeed (USB 2.0) access to the data is guaranteed using a dedicated storage controller which interfaces to the SD card on the StoneNode. The use of the mass-storage device class enables to connect the StoneNode to any computer, which highly improves its usability.

The StoneNode has been designed to be rugged and reliable. It contains only one PCB with no mechanically moving partsall components including the microSD card are soldered onto the PCB and the selected push buttons have a strong spring and software debouncing with a high time constant. The StoneNode is protected by a $5 \mathrm{~mm}$ thick metal housing (see Figure 1), and an additional epoxy fill ensures a firm attachment of the PCB and its components. For high reliability in the field, only industrial grade components have been selected, thereby guaranteeing an operating temperature range from -40 to $85^{\circ} \mathrm{C}$. More details on the component selection, hardware and firmware design are given in the following.

\section{A. Hardware}

As shown in Figure 2, the node is based on a MSP430 lowpower microcontroller and contains an integrated accelerometer and a gyroscope. Data is stored on a 2 GB microSD card which is soldered on-board in order to minimize the number of mechanical parts. In order to enable fast access to the microSD card, an additional data multiplexer has been inserted into the design which allows to mount the microSD card via a dedicated mass storage controller once the sensor node is connected to a computer. In normal operation, the microSD signals are routed to the microcontroller which accesses the card in the slower SPI mode. This still provides more than sufficient bandwidth to write the measured data, which amounts only to around $10 \mathrm{kB} / \mathrm{s}$. An UART to USB bridge provides additional debugging functionality, and 4 status LEDs, two general purpose push buttons and one reset button offer a minimal interface for the user to interact with the StoneNode.

TABle II. Selected Main Components and Details. The BAROMETER AND BuZzer are ONLY PRESENT ON STONENode v1.1

\begin{tabular}{|c|c|c|}
\hline Function & Part & Details \\
\hline MCU & TI MSP430FR5969 & $\sim 1220 \mu \mathrm{A} @ 3 \mathrm{~V}, 8 \mathrm{MHz}$ \\
\hline Accelerom. & ST H3LIS331DL & Triax $400 \mathrm{~g} 1 \mathrm{kHz} 12 \mathrm{bit}$ \\
\hline Gyroscope & InvenSense ITG-3701 & Triax $4000^{\circ} / \mathrm{s} 32 \mathrm{kHz} 16$ bit \\
\hline Storage & microSD $2 \mathrm{~GB}$ & SPI and SD interface \\
\hline Mux & TI TS3DV642 & Mux for SD signals \\
\hline USB IF & Microchip USB2641i & 3-port hub, mass-storage \\
\hline UART IF & FTDI FT230X & USB-UART bridge \\
\hline Supply & LiPo battery & Single-cell $3.7 \mathrm{~V} 1100 \mathrm{mAh}$ \\
\hline Charger & Microchip MCP73831 & LiPo charge management \\
\hline Barometer & MS5611-01BA03 & $10-1200 \mathrm{mbar} 100 \mathrm{~Hz} 24 \mathrm{bit}$ \\
\hline Buzzer & PKLCS1212E4001-R1 & $85 \mathrm{~dB}$ beep signal at $4 \mathrm{kHz}$ \\
\hline
\end{tabular}




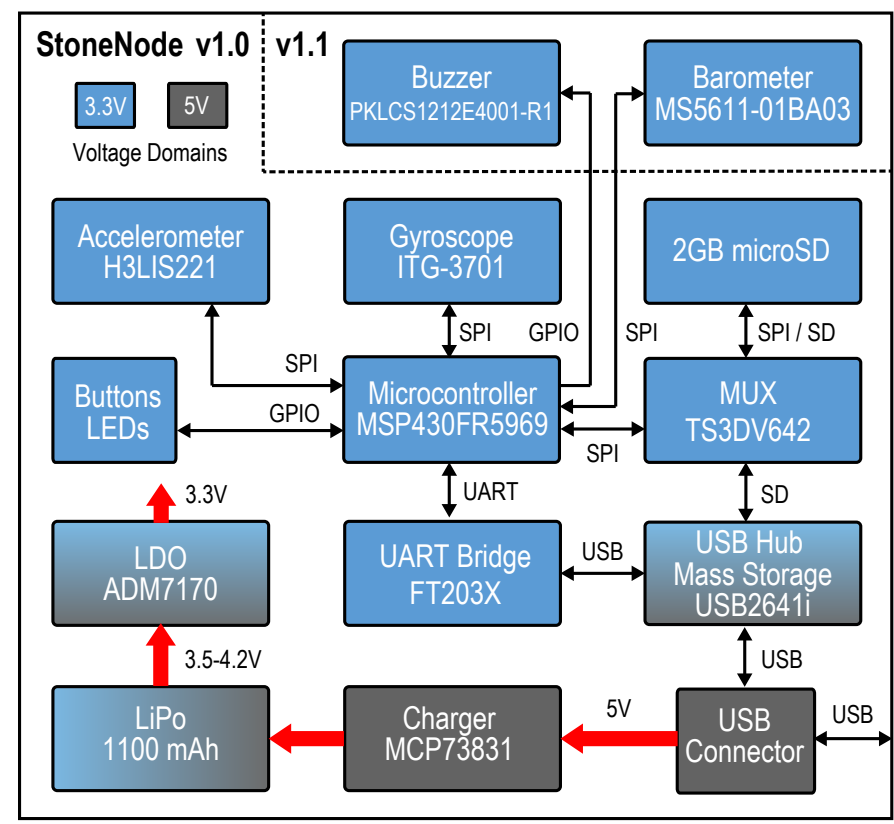

Fig. 2. Top-level block diagram of the StoneNode. The microcontroller is the core of the node, controlling the sensors, LEDs, buttons, USB interface and the microSD card. Besides an updated firmware and a more robust case, the StoneNode v1.1 contains an additional pressure sensor to measure altitude changes, and a buzzer to ease sensor retrieval in the field.

The node is powered from a LiPo battery which can be charged via USB. Components like the Mass Storage Controller, which are only required when the USB port is connected, are powered down during normal operation. Table II summarizes the main hardware components discussed in detail below.

Sensors Previous evaluations by the SLF elaborate the expected parameter ranges occurring in rockfall events [26]. For acceleration and rotational degrees of freedom the following sensor specifications are suggested: $0-500 \mathrm{~g}$ with $0.5 \mathrm{~g}$ resolution and sampling rates in the order of $1-10 \mathrm{kHz}$ for accelerations, and $6000 \% \mathrm{~s}$ with $90 \%$ sesolution and $10-100 \mathrm{~Hz}$ sampling rate for angular velocities. We aim at fulfilling these specifications with completely integrated, tri-axial MEMS sensors. While there exists a broad variety of such devices on the market today, most of them only cover a low dynamic range (typically less than $10 \mathrm{~g}$ in case of accelerometers) as these sensors were primarily developed for consumer electronic devices such as cellphones. There are only a few sensors available which reach the levels required by the suggested specifications above. In order to guarantee high resolution data sampling for the key internal parameters we restricted the initial design to hosting the accelerometer H3LIS331DL from STMicroelectronics, which covers a dynamic range of $\pm 400 \mathrm{~g}$ with a resolution of 12 bit $(0.195 \mathrm{~g})$ at a maximum sampling rate of $1 \mathrm{kHz}$ and the ITG-3701 gyroscope from Invensense offering $\pm 4000 \%$ s with a high resolution of 16 bit $(0.122 \%$ s $)$ and a maximum sampling rate of $32 \mathrm{kHz}$.

StoneNode v1.1 hosts additionally a MS5611-01BA03 sensor from MEAS, which is a new generation altimeter sensor with an altitude resolution of up to $10 \mathrm{~cm}$ using an over- sampling rate of 4096. However, as this results in a long response time of almost $9 \mathrm{~ms}$, we are currently using a lower oversampling rate of 512 with a response time around $1.1 \mathrm{~ms}$ and an effective pressure resolution around $0.042 \mathrm{mbar}(\approx$ $0.4 \mathrm{~m}$ at sea level) in order to have more headroom in the polling mechanism of the firmware. The pressure values are currently sampled at $100 \mathrm{~Hz}$, together with additional temperature measurements provided by the sensor for linearization temperature compensation.

Microcontroller The microcontroller periodically fetches the data from the two sensors and stores them in the non-volatile memory. It also steers all circuit blocks with control signals, handles all user inputs and manages visual notifications using LEDs. It further provides a simple UART command-line interface for debugging purposes. The MSP430FR5969 from Texas Instruments has been selected for its low power consumption of $\sim 3.6 \mathrm{~mW}$ at $3 \mathrm{~V}$ and $8 \mathrm{MHz}$ in active, and $\sim 1.2 \mu \mathrm{W}$ in standby mode. The internal peripherals allow for convenient integration with the sensors and the memory with a minimal amount of additional external components.

Interfaces In order to make the hardware interface as simple as possible, the sensor node has been designed such that all required functionality is possible with only one Micro USB connector on board. The USB interface is used for high-speed access to the measured data, charging the internal battery and communicating with the microcontroller via UART. To allow the users to download the data in a plug-and-play manner, a USB hub chip which supports the proprietary SD card interface natively and provides another downstream USB port for the USB to UART interface has been selected. More in detail, the Microchip's USB2641 has been used in our design, so the flash memory card on the StoneNode is recognized as a regular mass-storage device on the host PC side. On the microcontroller side an FT230X USB to UART bridge provides additional debugging support. In order to route the SD card signals to either the microcontroller or the USB hub chip, we chose the high-speed signal multiplexer TS3DV642 from Texas Instruments.

In order to facilitate retrieval of the sensor nodes in the field, we added a buzzer (PKLCS1212E4001-R1) to the StoneNode v1.1 that starts emitting a $85 \mathrm{~dB} 4 \mathrm{kHz}$ beep signal after a user programmable timeout (currently set to $15 \mathrm{~min}$ ). The timeout is needed to prevent the generated vibrations and pressure oscillations from corrupting the main measurements. We also record a time stamp on the SD card in order to keep track of the time instant the buzzer has been activated.

Power Supplies When developing a battery-powered device for mobile usage, the power consumption is always a crucial issue. There is a trade-off between battery size and operating duration. To maximize the latter while minimizing the former, special care has to be taken on the power consumption of all involved components. Since all parts are operated at $3.3 \mathrm{~V}$, there are no losses due to derivation of multiple supply voltage rails. Nevertheless, the battery voltage of $3.5 \mathrm{~V}$ to $4.2 \mathrm{~V}$ has to be brought to the desired value of $3.3 \mathrm{~V}$. Although the node should be low power, stable supply rails with low noise are desirable 

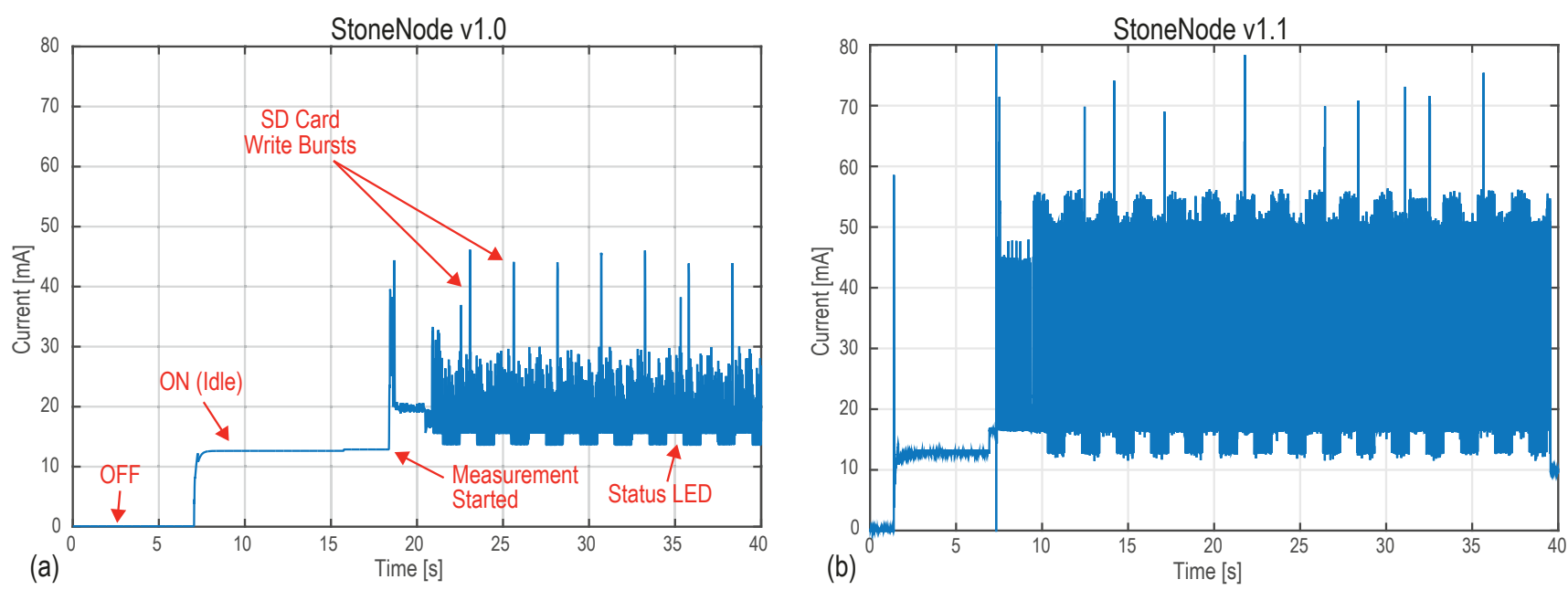

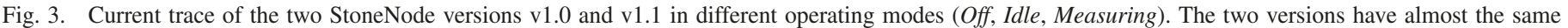

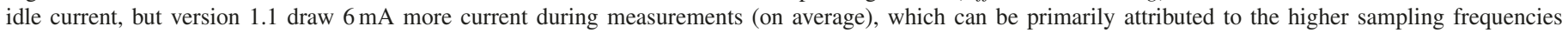
of the sensors, which in turn produces more data to be stored on the SD card.

in order to ensure reliable sensor operation and accurate data acquisition. Therefore, a low-dropout linear regulator has been selected for the high stability of its output voltage. The choice fell on the ADM7170 from Analog Devices which features a low dropout voltage of $42 \mathrm{mV}$ and very low noise levels $(5 \mu \mathrm{V}$ RMS). Since the battery is non-removable and mounted in the case (Figure 1), an on-board battery charger is required. In general, a LiPo battery must be charged by following a controlled profile with different charging states. There exist many specialized ICs to fulfill this task. We selected the MCP73831T-2ACI from Microchip, which is an integrated single-cell LiPo charge management controller. Many features like charge termination voltage, end-of-charge control and preconditioning charge current are set in hardware.

\section{B. Firmware}

The firmware has been written to manage the data generated by the sensors and store the data in a reliable way. To achieve the best energy efficiency, the accelerometer and gyroscope interrupt the microcontroller after a sample has been taken. The barometer and the temperature sensor contained in the barometer do not support interrupts and are therefore being polled in the interrupt routine after servicing the other two sensors. Each time a sample is read from a sensor, a time stamp is generated and combined with the data collected from the sensor which triggered the interrupt. This data is saved temporarily in a FIFO buffer in the microcontroller. Once $512 \mathrm{~B}$ of sensor data has been collected, the buffer is flushed to the SD card in one burst. The internal digital clock (DCO) generates the main $8 \mathrm{MHz}$ clock of the microcontroller.

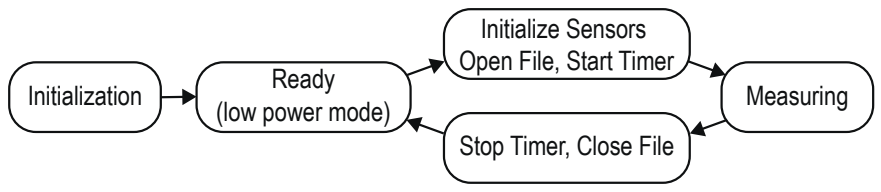

Fig. 4. Visualization of the main functions of the firmware.
Figure 4 shows a flow chart of the main firmware functionality. After the initialization phase the system goes into ultra low power mode and waits for an interrupt from the user buttons. When the 'measuring' phase starts, the data from the sensor is acquired and stored in the SD card flash memory by using the FAT file system [31]. For each measurement series, a new file is created with consecutive sequence number. Each file contains a 32 bit header, containing information on the sampling rate and the range of the sensors. In addition, the file contains a CRC checksum at the end, which is generated using the dedicated hardware module available on the MSP430 microcontroller. This checksum can be used to verify data integrity during sensor readout, and prevent corrupted measurements to enter further down-stream analyses of the sensor data.

To conserve microcontroller resources, the barometer linearization and temperature compensation is not performed at run-time, but postponed to a post-processing step after sensor readout. Hence, the calibration parameters are read from the factory programmed ROM in the sensor initialization phase, and stored to the measurement file.

\section{IV.EXPERIMENTAL RESULTS}

In this section, we first present power measurements of both StoneNode versions. Then, we explain how the sensors were calibrated and provide several measurements gathered from induced rockfall experiments in the field. We discuss the obtained measurement traces and provide first results and insights from simulations where the acquired experimental data has been used to calibrate the RAMMS::ROCKFALL module.

\section{A. Power Consumption}

Measurement traces of the current consumption of both StoneNodes are shown in Figure 3, and have been obtained by operating the devices in unattached mode (i.e. no USB connection) with a fully charged battery $(4.2 \mathrm{~V}, 1100 \mathrm{mAh})$.

The quiescent current of the StoneNode v1.0 is around $83 \mu \mathrm{A}$ and is composed of the supply current of the LTC2950 

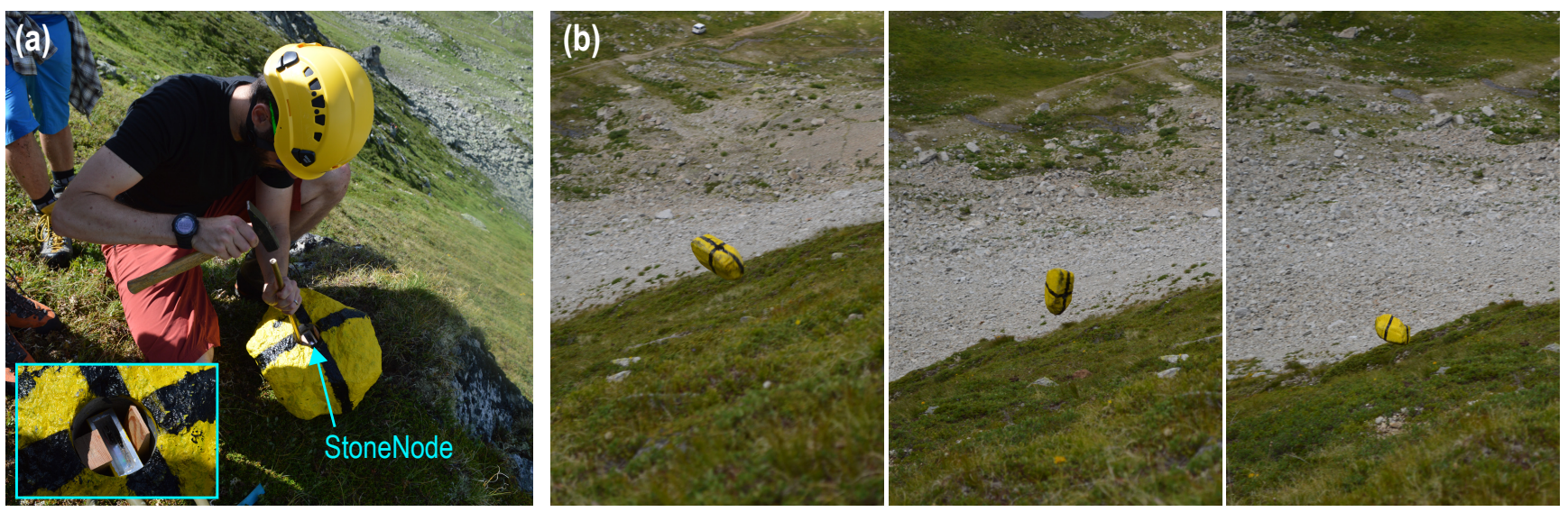

Fig. 5. (a) The StoneNodes are mounted in a $68 \mathrm{~mm}$ hole drilled into the specimen rocks using wooden wedges. (b) StoneNodes in action.

ON/OFF power controller drawing around $10 \mu \mathrm{A}$, the reverse leakage of the battery charger of around $10 \mu \mathrm{A}$ and finally due to leakage of the voltage divider for measuring the battery voltage $(21 \mu \mathrm{A})$ and the pull-up resistor for of the LTC2950's open drain output $(42 \mu \mathrm{A})$. The idle current (ON but not measuring) is around $12.7 \mathrm{~mA}$ with activated power LED and $8.8 \mathrm{~mA}$ without it. The difference matches the LED current of around $4 \mathrm{~mA}$. The main contribution to the idle current is the operating current of the main power supply's LDO with around $5 \mathrm{~mA}$ (worst case).

The first peak at $18 \mathrm{~s}$ in Figure 3a) occurs during the card initialization phase of the first measurement. During logging, the different short peaks show the activity of the SD card, which in the current configuration $(10 \mathrm{kB} / \mathrm{s}$ in $512 \mathrm{~B}$ bursts) is accessed every $50 \mathrm{~ms}$. Some of these peaks are significantly higher than the remaining ones, which can be explained with internal storage operations of the microSD card. The repetitive duty cycle pattern where the current consumption jumps in $2 \mathrm{~mA}$ steps is due to the activity LED indicating that a measurement is in progress.

The average current consumption during a measurement is around $17.5 \mathrm{~mA}$ with activated LEDs, corresponding to a power consumption of $73.5 \mathrm{~mW}$. Turning off the LEDs constantly would reduce the current and power consumption

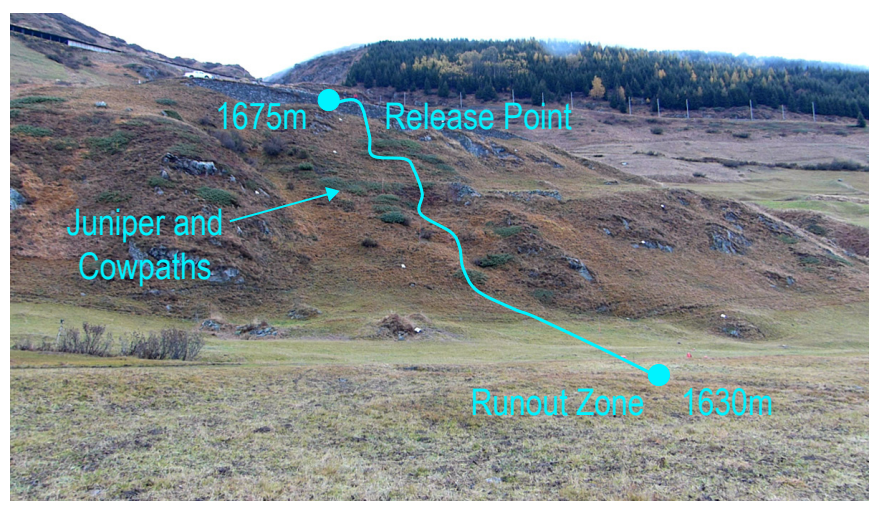

Fig. 6. Overview of the test site located in the Oberalp pass region in the Swiss Alps. The trace indicates a possible rockfall trajectory. to $12.6 \mathrm{~mA}$ and $53 \mathrm{~mW}$, respectively. Interestingly, with the relatively low write data rate of $10 \mathrm{kB} / \mathrm{s}$, the SD card has an average current consumption of only $5 \mathrm{~mA}$, which is significantly less than the maximum value of $200 \mathrm{~mA}$ specified for the high-speed mode in the SD standard [33].

In Figure 3b) we can see that the revised StoneNode v1.1 has almost identical idle current $(12.85 \mathrm{~mA}$ instead of $12.7 \mathrm{~mA}$ ), but the higher sampling rate of the sensor results in a current draw of $23.5 \mathrm{~mA}$ during measurements, on average, which is $6 \mathrm{~mA}$ more than with v1.0.

Long-time tests showed that the limiting factor is not power consumption (which theoretically would provide more than 60 operating hours with one battery charge for the StoneNode v1.0), but the capacity of the 2 GB SD card. With current data rates of $10 \mathrm{kB} / \mathrm{s}$ and $27 \mathrm{kB} / \mathrm{s}$ for the StoneNode versions 1.0 and 1.1 , this results in operating times of around $56 \mathrm{~h}$ and $20.5 \mathrm{~h}$, respectively. The current battery and memory provisioning of the StoneNode hence enables uninterrupted field tests lasting for several working days.

\section{B. Sensor Calibration}

To compensate for any sensor offsets, we developed a calibration setup based on a rotating disk whose rotational velocity can be precisely controlled. The StoneNode to be calibrated is mounted at an off-axis position on that disk, and the rotational speed and centripetal forces are measured for a different speed settings ranging from $500 \%$ to $3000 \%$ s. The process is repeated for all three sensor axes, and a linear correction function $f(x)=c_{0}+c_{1} \cdot x$ is computed for each sensor axis. The dominant correction term for the accelerometer is the constant offset $c_{0}$ and its value is in the order of $0.15 \mathrm{~g}$ to $3 \mathrm{~g}$. In case of the gyroscopes, the proportional term $c_{1}$ is dominant and shows deviations in the order of $0.09 \%$ to $0.35 \%$ from the ideal value of 1.0 .

\section{Field Tests}

Besides controlled lab experiments described in the sensor calibration routine, we have conducted several field tests. The experiment described in the following is a classical induced rock fall experiment that has been carried out in the Swiss Alps to gather statistics of the deposition locations, and several ac- 

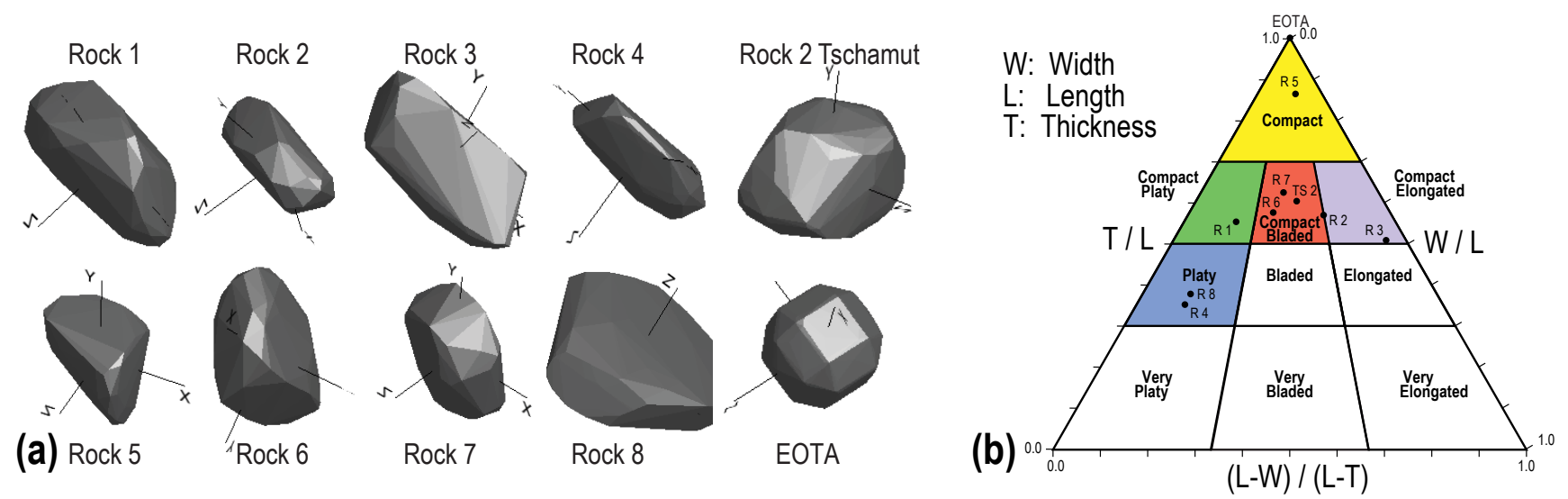

Fig. 7. (a) Visualization of the digitized models of the instrumented rocks (called Bergell 1-8) and the additional Tschamut 2 and EOTA boulders used in RAMMS::ROCKFALL. (b) Sneed and Folk [32] classification diagram (elongation and flatness) of the instrumented rocks. The dimensions, mass and volume of the rocks are summarized in Table III.

celeration and rotational velocity traces for calibration and validation of the RAMMS::ROCKFALL module (Section IV-E).

Note that the measurements presented here have been acquired in autumn 2016 with the StoneNode v1.0. The StoneNode v1.1 has been developed during the winter period 2016/2017 and due to the Swiss Alpine weather conditions and snowfall it could not yet be tested in the field. However, initial tests in the lab show that all functions work correctly, and we currently plan to deploy the updated sensors in further field tests during the summer period 2017.

Experimental Setup In the following, we present results from a larger experiment on a testing site with grassy terrain interspersed with stones (depicted in Figure 6). The terrain has a slope of $>30^{\circ}$ which is typical for areas with increased rockfall risk. The rock falls have been manually induced by releasing differently shaped boulders with $30 \mathrm{~kg}-80 \mathrm{~kg}$ of mass at the Release Point shown in Figure 6. The StoneNode is mounted within the boulder to be measured by wedging it into a drilled hole located at the center of mass as shown in Figure 5a). This ensures accurate tracking of the rock rotations, and protects the sensor from damage. Falling rocks in action are depicted in Figure 5b).

The properties of the rocks used in this experiment are

TABLE III. SPECIFICATIONS OF THE GRANITE ROCKS (BERGELL Rock 1-8) and Two AdDitionally Used Rocks (a HeAVy Rock NAmed Tschamut 2 and the ARTIFICIAL EOTA TeSt Rock). THE Rocks Have BeEn Instrumented With StoneNodes.

\begin{tabular}{lccc}
\hline Rock & Dimensions $(\mathrm{m})$ & Weight $(\mathrm{kg})$ & Volume $\left(\mathrm{m}^{3}\right)$ \\
\hline 1 & $0.36 / 0.32 / 0.20$ & 30.5 & 0.011 \\
2 & $0.41 / 0.30 / 0.24$ & 34.8 & 0.013 \\
3 & $0.49 / 0.25 / 0.27$ & 42.4 & 0.016 \\
4 & $0.43 / 0.48 / 0.18$ & 34.8 & 0.012 \\
5 & $0.37 / 0.34 / 0.32$ & 42.6 & 0.016 \\
6 & $0.45 / 0.37 / 0.26$ & 41.1 & 0.016 \\
7 & $0.33 / 0.40 / 0.25$ & 29.9 & 0.011 \\
8 & $0.52 / 0.58 / 0.22$ & 52.2 & 0.019 \\
TS2 & $0.50 / 0.39 / 0.30$ & 78.4 & 0.030 \\
EOTA & $0.30 / 0.30 / 0.30$ & 44.0 & 0.016 \\
\hline
\end{tabular}

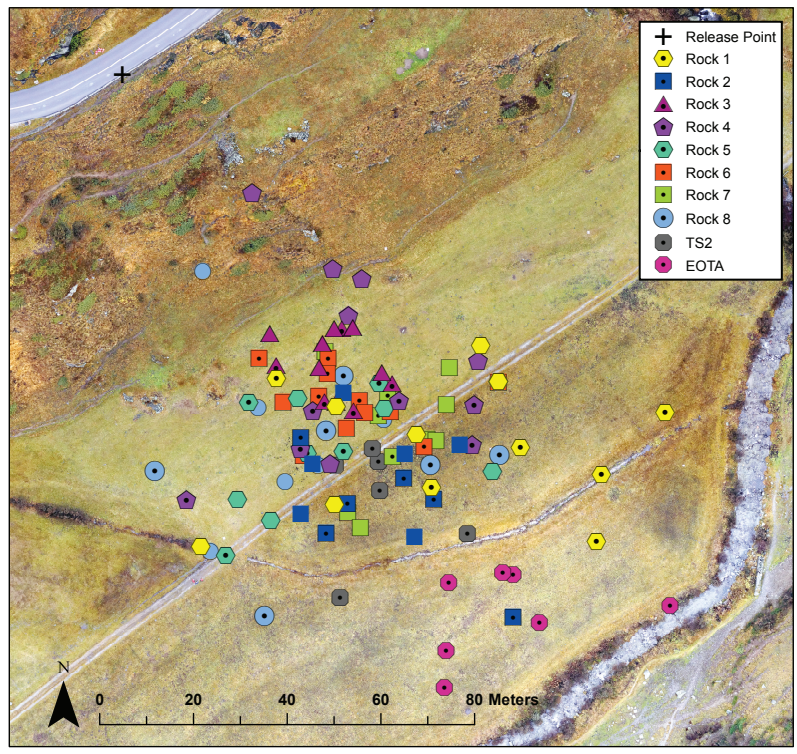

Fig. 8. Deposition distribution of the Rocks 1-8, TS2, and EOTA. The dotted markers represent the deposition points recorded under frozen terrain conditions.

listed in Table III, and the corresponding digital models that are used later on in RAMMS::ROCKFALL are depicted in Figure 7a). As can be seen in the Sneed and Folk diagram [32] in Figure $7 b$ ), these rocks span several different morphological categories. The rocks have been released each under wet and frozen terrain conditions 6-7 times in sequence in order to gather several measurement traces of the same rocks (some of the boulders disintegrated during the experiment and could not be released anymore).

Deposition Distribution and Measurements The deposition distribution of the Bergell Rocks 1-8 as well as for the TS2 block and a perfectly symmetric EOTA (norm rock of the European Organisation for Technical Assessment used in standardized rock fence testing procedures in official European Technical Approval Guidelines) block are depicted in Figure 8, and several typical accelerations and rotations that have been 

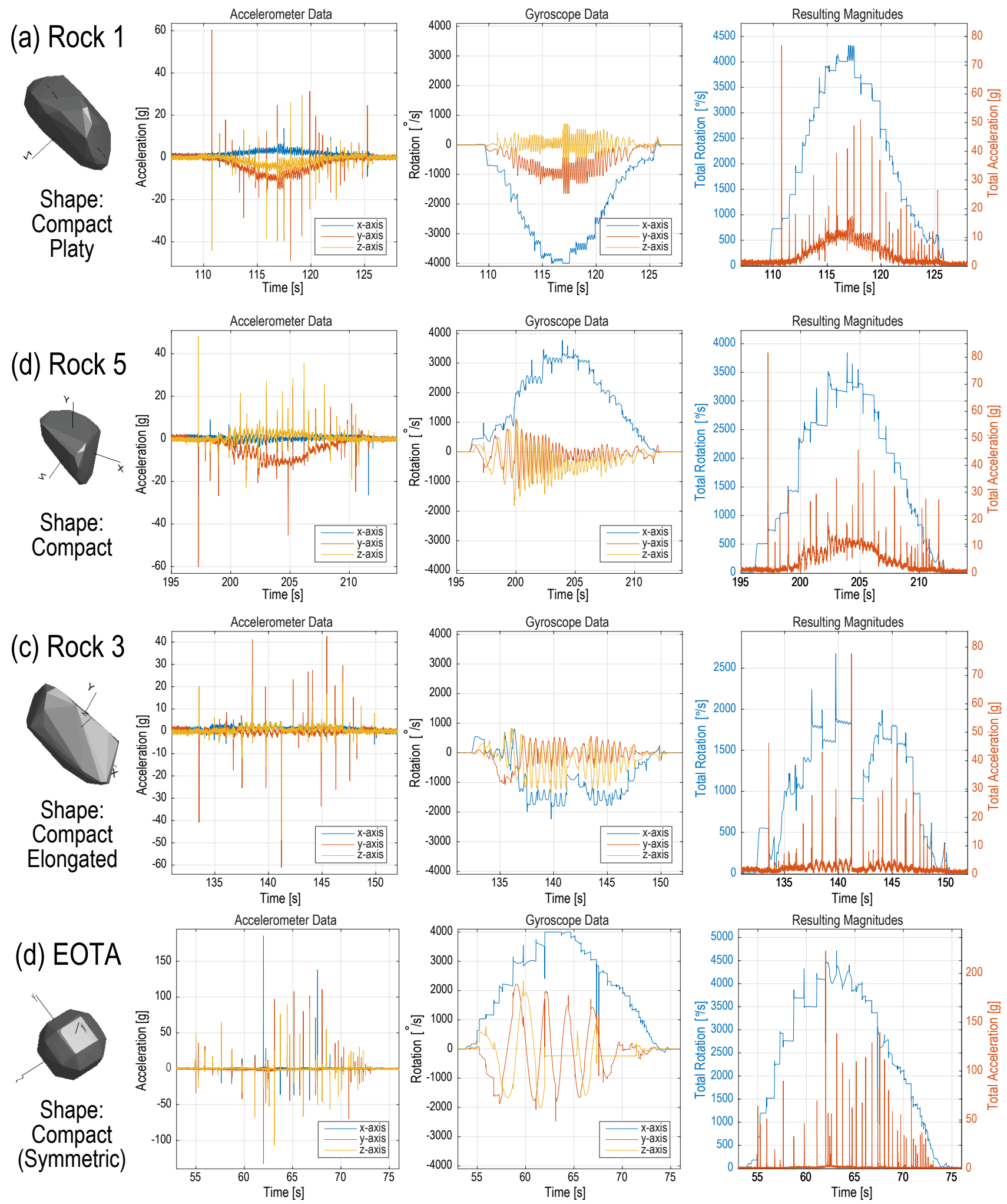

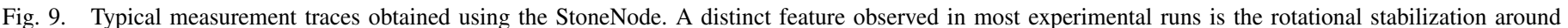

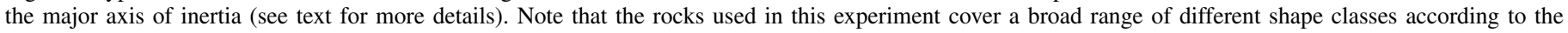
morphological classification by Sneed and Folk shown in Figure 7. 
(a)

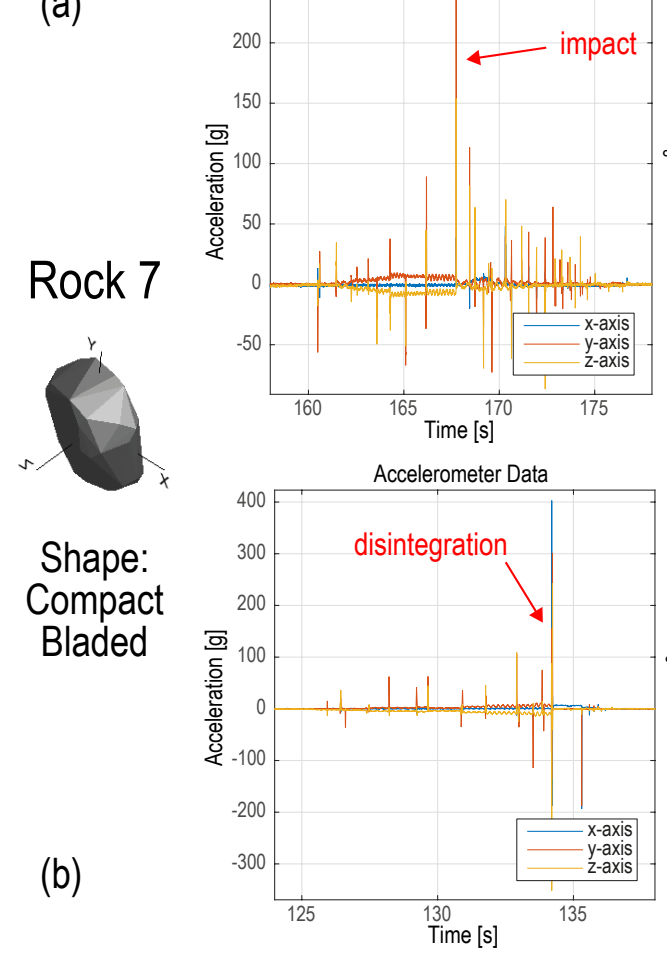

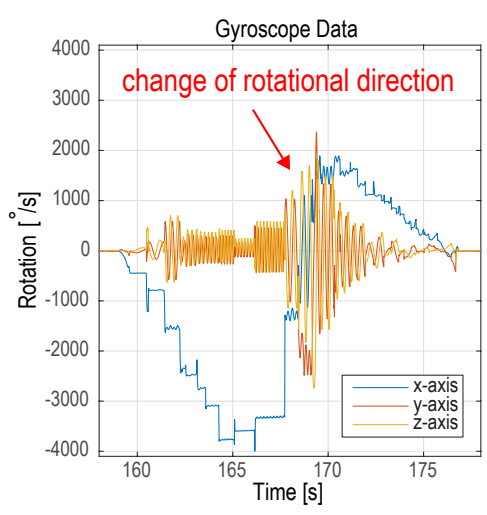
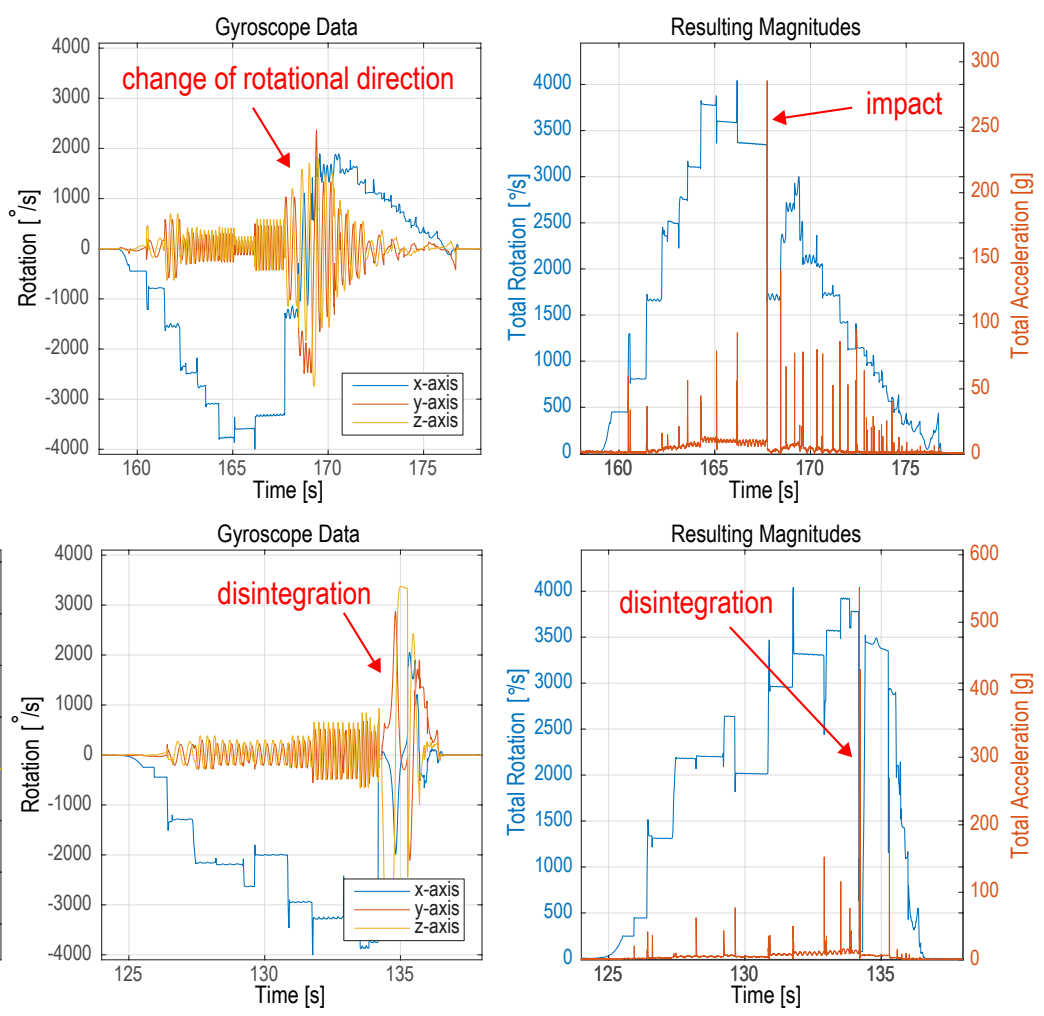

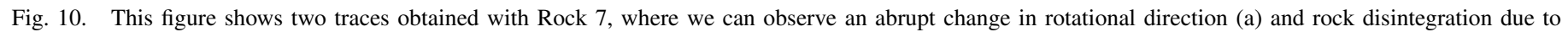
a heavy impact on the ground (b).

recorded with the StoneNodes are shown in Figures 9 and 10.

A predominant feature of most of the recorded measurements shown in Figure 9 is the stabilization of the rotation around the largest axis of inertia. This is not only the case for platy, wheel shaped boulders such as Rock 1 in Figure 9a), but holds equally so for more compactly shaped rocks such as Rock 5 in Figure 9b). Obviously, the rotation amplitude becomes larger the closer the rock shape is to an ideal wheel shape. Only heavily elongated rocks diverge from this distinct rotational behaviour as can be seen in the measurement of Rock 3 in Figure 9c). The EOTA shape promises to be more susceptible to small perturbations. In the experiment, the breaking of the symmetry by the sensor mounting hole resulted equally to a stable rotation around its major axis of inertia, as can be seen in Figure 9d). The main difference with respect to the rotational features exhibited by compact, natural rocks (such as Rock 1) are the pronounced, slower rotations around the two minor axis of rotation.

Apart from rotational stabilization, we also observed other interesting features in this experiment such as abrupt changes of rotational direction due to heavy impacts on the ground as depicted in Figure 10a), or the destruction of the rock which is visible as a $550 \mathrm{~g}$ resultant acceleration peak around the $134 \mathrm{~s}$ mark in Figure 10b). Note that the sensor remained fully functional after disintegration of Rock 7.

Practical Experience Gained with the StoneNode The StoneNodes used in these field tests have been intensely strained and they survived more than 50 tests with several heavy impacts $(>400 g)$ and disintegrating boulders, and are still working. Only two immediate improvements were evident: first, the case should have a lid since the sunk-in mounting position does not provide sufficient protection of the USB port and buttons. And second, the node should have an acoustic signal emitter in order to facilitate sensor node retrieval in cases where the specimen boulders disintegrated. These improvements have been already implemented in the StoneNode v1.1, as described in Section III.

The observed rotational velocities for small rocks just exhaust the range limit of $4000 \%$ per axis of the deployed sensors. However, since we plan to rather move towards larger rocks in future experiments, the current conditions represent the extreme case. Larger rocks will have a larger moment of inertia, and are hence expected to exhibit lower rotational speeds in such experiments. Further, acceleration peaks above the per axis limits of $400 \mathrm{~g}$ of the sensors only occurred in rare special cases, e.g., when the boulder broke apart. Therefore, there are no immediate plans to increase the range of the accelerometer and gyroscope. However, we decided to increase the sampling rate from $400 \mathrm{~Hz}$ and $500 \mathrm{~Hz}$, respectively, to $1 \mathrm{kHz}$ for higher temporal resolution in the StoneNode v1.1 to track the impact dynamics in greater detail.

\section{Digital Elevation Model}

In order to perform post-experimental parameter evaluations in RAMMS::ROCKFALL using the data acquired with the StoneNodes, a highly accurate digital elevation model (DEM) 

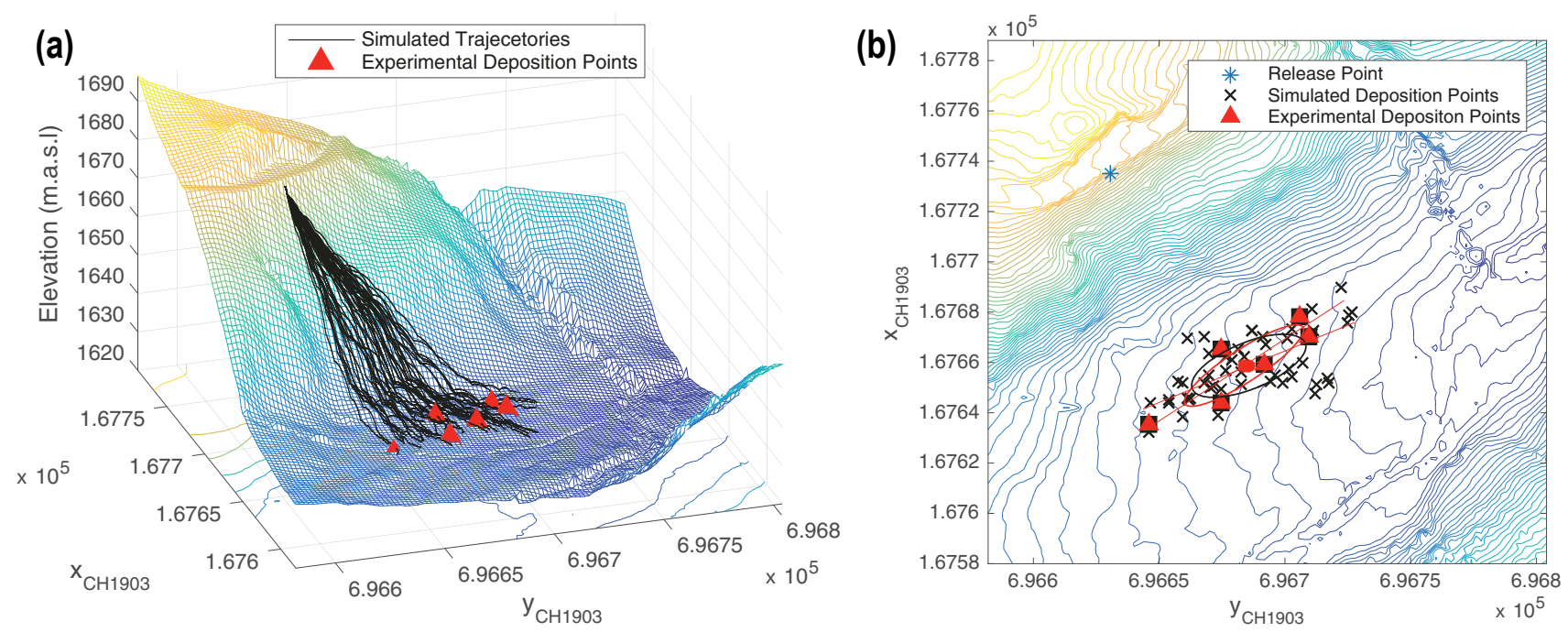

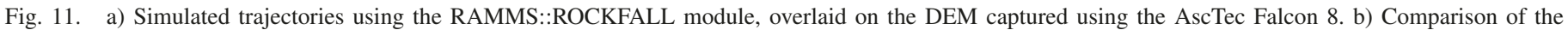
experimental deposition distribution and the simulated one after calibration of the simulation module.

is required. Over the last years laser scanning and aerial photogrammetry by unmanned aerial systems (UAS) have been the tools of choice. Alpine applications most often feature rugged and impeded access to the experimental site. Thus, a light and transportable system is favorable. For the DEM used in this paper, an Ascending Technologies (AscTec) Falcon 8 Octocopter equipped with an externally modified Sony Alpha NEX-7 camera is deployed. The full weight of the system totals to $2.3 \mathrm{~kg}$ (incl. camera) and can be transported to remote locations fully assembled in a special backpack. The Sony Alpha NEX-7 system camera uses a 24 MPixel APSC CMOS sensor alongside with a Sony NEX 20mm F/2.8 optical lens. Mounting point at the Falcon 8 is a gimbal featuring active stabilization and vibration damping powered by the UAS battery. Simultaneously to the UAS data acquisition, roughly 10 different reference heights have been measured within the test site with a Trimble GeoXH differential GNSS with an accuracy of $10 \mathrm{~cm}$.

The required dimensions of the DEM determines the flight path, where sufficient spatial overlap has to be allocated in the individual pictures for post-processing. The obtained UAS imagery was processed using AgiSoft PhotoScan Pro v1.2.4, a commercial software extensively used in the UAS community. For the absolute orientation the recorded ground control points are used. The DEM then can be exported in different resolutions via the the PhotoScan interpolation algorithm. Usually, DEM resolution is reduced to two meter grid size as concession to generally available DEM resolution for practitioners. However, a DEM down to a grid size of $5 \mathrm{~cm}$ is possible with the described acquisition and post-processing (see [34] for more details on DEM generation using UASs).

\section{E. Preliminary RAMMS::ROCKFALL Calibration}

As opposed to most other rockfall simulation programs, RAMMS::ROCKFALL is not governed by restitution coefficients, that is by simple rebound behaviour of a point mass, but rather by the slippage dependent friction law presented in [7]. The rolling, and above all, stick-slip motion happening at every impact is described by

$$
\mu(s)=\mu_{\min }+\frac{2}{\pi}\left(\mu_{\max }-\mu_{\min }\right) \arctan (\kappa s)
$$

where the effective friction $\mu(s)$ evolves from a smaller $\mu_{m i n}$ to a higher $\mu_{\max }$ during the contact time governed by a time scale $\kappa$. Here, the slipping distance $s$ is the distance which the center of mass of the rock travels during the contact phase. The temporal evolution of the slipping distance is assumed to decay to zero when the rock is no longer in contact with the terrain, that is

$$
\dot{s}=\beta s
$$

In contrast to most other rockfall simulation software the only stochastic process in RAMMS::ROCKFALL is the randomized start position of the released block. The subsequent trajectory path is completely determined via Newtonian laws and the numerical scheme of non-smooth contact dynamics description together with hard contact laws.

A first step towards improving the calibration of the RAMMS::ROCKFALL simulation module has been performed by using the experimental data to find the friction parameters for a given terrain. The calibration is currently done by sweeping these friction parameters $\mu_{\text {min }}, \mu_{\max }, \kappa$ and $\beta$ alongside with a velocity dependent drag force $d$ to account for additional energy dissipation during the contact time over their range of interest, and comparing the rock trajectories and deposition points of the resulting simulations with the data obtained from the experiments. This involves on one hand the final deposition distribution visible in Figure 8, and on the other hand the measured angular velocities of the rocks-which is an important property that can be used to screen the simulation runs against unrealistic behaviours. This is, we measure the congruence of the simulation and experimental data using the center of mass offset and the major axis angle offset between the Gaussian ellipsoids fitted to the deposition distributions. 

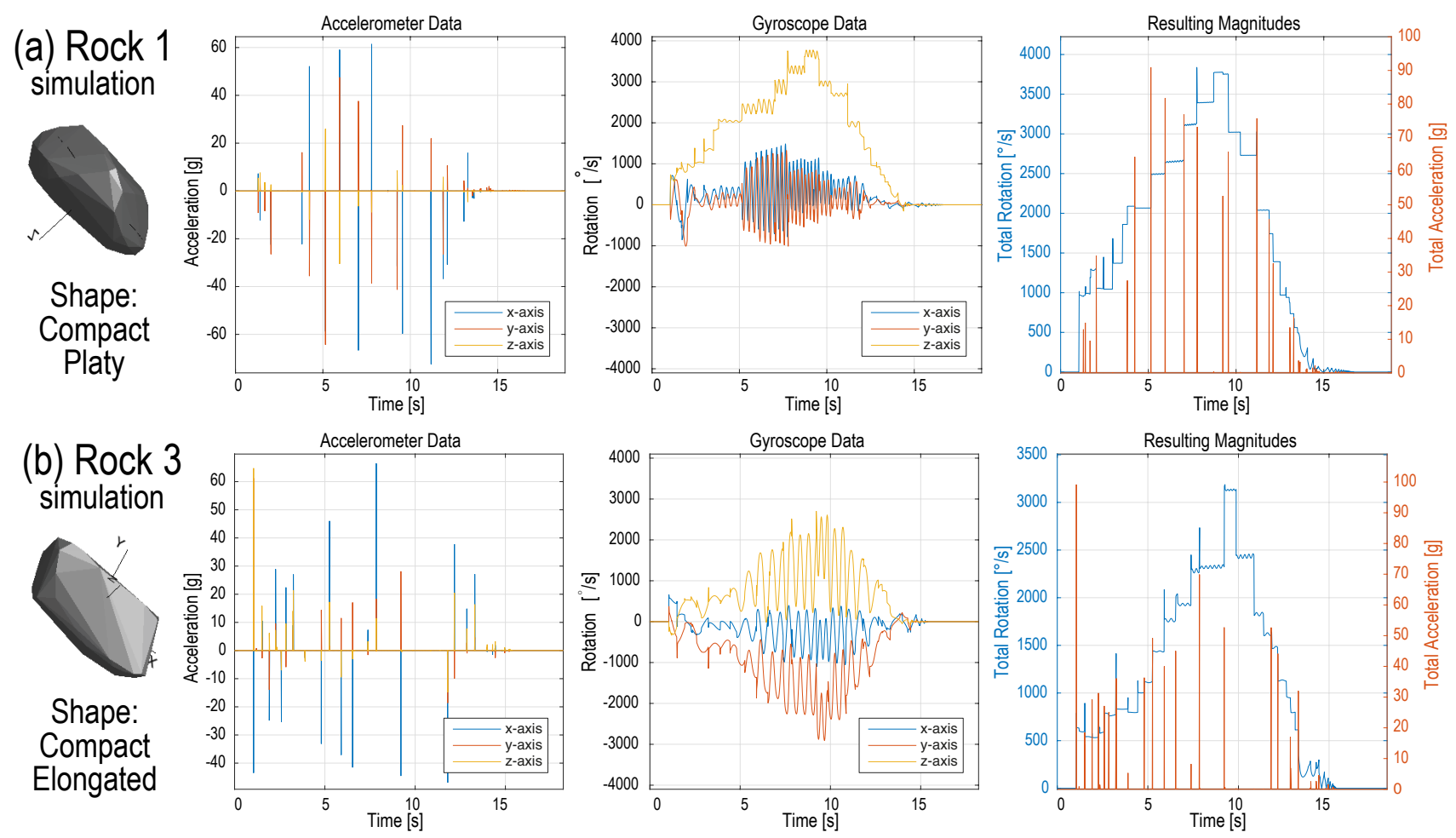

Fig. 12. Instances of simulated accelerations and angular velocities for Rock 1 and Rock 3. Note that when comparing these traces to the experimentally obtained ones in Figure 9, we can observe that these traces exhibit both quantitatively and qualitatively similar motion patterns.

Further, as platy shaped rocks exhibit most prominently the rotation around their largest axis of inertia, for those rocks a lower boundary of $1000 \% \mathrm{~s}$ on the mean angular velocity is set as exclusion criterion. Data sets with lower overall rotation are discarded. Future calibration might extend these routine to more sophisticated exclusion rules, especially including thresholds on all axis.

Figure 11 shows the results of this calibration for the experiments conducted exemplary for Rock 1 . Subfigure a) visualizes the simulated traces together with the 3D DEM used by the RAMMS simulation module, and subfigure b) shows a comparison of the calibrated simulation run, together with the experimental data. We can observe good qualitative and quantitative agreement between the experimental data and the simulated traces. For most rocks the center of mass offset criterion can be fulfilled within less then $0.5 \mathrm{~m}$ agreement. In order to have several parameter sets for comparison with the other rocks this criterion is softened such that a minimum of 10 sets remain in the pool. This is achieved in increasing the precision level of the offset by half percent steps of the travelled distance. For Rock 1, this yields 18 parameter sets having a matching center of mass within a $0.92 \mathrm{~m}$ radius around the experimental deposition center. For these sets, the alignment of the main axis of the normal distribution matches between 0.1 and 18 degrees. The same routine is applied for all rock shapes yielding parameter sets $p_{R 1}, p_{R 1}, \cdots, p_{R 8}$ and the numbers of sets and congruence lies in the same order of magnitude for all of them. Finally, the $p_{R 1}$ to $p_{R 8}$ are searched for overlapping sets. Here, the best set is chosen to be the friction law with most appearances as an entire set in $p_{R 1}$ to $p_{R 8}$. The best fitting parameter set is found to appear in $p_{R 3}, p_{R 4}, p_{R 5}$, and $p_{R 7}$ while $p_{R 1}, p_{R 2}$, and $p_{R 6}$, feature sets only differing in a single parameter from the best fit, or two parameters for $p_{R 8}$, respectively.

Finally, Figure 12 shows simulated accelerations and rotational velocities for Rock 1 and Rock 3, after calibration of the simulation model. When comparing these traces to the experimentally obtained ones in Figure 9, we can observe that they exhibit both quantitatively and qualitatively similar motion patterns.

\section{Conclusions}

We presented StoneNode v1.1, a rugged low-power sensor device for the acquisition of rockfall dynamics in induced experiments which are vital ingredients for the development of accurate simulation models. The node employs low-power MEMS sensors with high dynamic range with up to $1 \mathrm{kHz}$ sampling rate. The StoneNode has been tested in-field during several test in the Swiss Alps and the data that has been acquired with several rocks has been used to calibrate and validate a state of the art simulation tool. Detailed results from the field tests and post-processing steps have been presented in this paper and they confirm that the node is convenient and easy to use. The calibration methodology employed is still relatively simple and we expect to be able to further enhance the simulation performance by carrying out more elaborate analyses with the experimental data traces, gaining insights into the different behavior caused by varying boundary 
TABLE IV. Final Specs of the StoneNode V1.0 AND v1.1.

\begin{tabular}{ll}
\hline Property & Value/Range \\
\hline Main Interface & USB 2.0 (mass storage, UART, power) \\
Storage Medium & 2 GB microSD card \\
Accel. Resolution & $\pm 400 \mathrm{~g}, 12 \mathrm{bit}, 400 \mathrm{~Hz}^{\dagger}, 1 \mathrm{kHz}^{\ddagger}$ \\
Gyro Resolution & $\pm 4000^{\circ} / \mathrm{s}, 16 \mathrm{bit}, 500 \mathrm{~Hz}^{\dagger}, 1 \mathrm{kHz}^{\ddagger}$ \\
Operating Range & $-10^{\circ} \mathrm{C}$ to $40^{\circ} \mathrm{C}\left(-40^{\circ} \mathrm{C} \text { to } 85^{\circ} \mathrm{C}\right)^{\S}$ \\
Barometer & $10-1200 \mathrm{mbar}, 24$ bit, $100 \mathrm{~Hz}$ \\
Max. Logging Time & $56 \mathrm{~h}^{\dagger}, 20.5 \mathrm{~h}^{\ddagger}$ (limited by $2 \mathrm{~GB}$ storage) \\
Dimensions & $62 \times 52 \times 27 \mathrm{~mm}$ (with case) \\
Material Cost & $\sim 180 \mathrm{US} \$^{\dagger}$ and $\sim 195 \mathrm{US} \$^{\ddagger}$ (with case) \\
\hline
\end{tabular}

${ }^{\S}$ range without battery, ${ }^{\dagger}$ StoneNode v1.0 ${ }^{\ddagger}$ StoneNode v1.1

conditions. Additionally, equivalent experiments with larger rocks are planned in order to investigate whether it is possible to unify rock models and come up with generic models that are independent of rock size and shape. This methodology allows for a consistent (re-)calibration of the simulation model, which is of major importance when applying software patches to the simulation kernel since it is expected that subsequent software releases behave similarly.

In the long run, we aim at reconstructing complete trajectories with sensor fusion approaches similar to the ones outlined in early studies by the SLF [26], possibly also employing other sensor systems such as low-power LPS or GPS systems. The revised StoneNode v1.1 represents a next step towards this goal from the side of the acquisition device, since it offers higher sampling rates and an additional pressure sensor which can be used to capture additional information.

\section{ACKNOWLEDGMENTS}

We would like to thank Tim Aebi, Thomas Birchler and Jonas Aeschbacher for their commitment and firmware work during their semester project.

\section{REFERENCES}

[1] N. Hilker, A. Badoux, and C. Hegg, "Unwetterschäden in der Schweiz im Jahre 2006," Wasser Energie Luft, 2007.

[2] T. Heckmann, L. Hilger et al., "Integrating field measurements, a geomorphological map and stochastic modelling to estimate the spatially distributed rockfall sediment budget of the upper kaunertal, austrian central alps," Geomorphology, vol. 260, pp. 16-31, 2016.

[3] G. Gigli, S. Morelli et al., "Terrestrial laser scanner and geomechanical surveys for the rapid evaluation of rock fall susceptibility scenarios," Landslides, vol. 11, no. 1, pp. 1-14, 2014.

[4] E. Hoek, "Analysis of rockfall hazards," Rock Engineering, 2006.

[5] A. D. Regmi, P. Cui et al., "Rock fall hazard and risk assessment along araniko highway, central nepal himalaya," Environmental Earth Sciences, vol. 75, no. 14, p. 1112, 2016.

[6] M. Christen, Y. Bühler et al., "Integral Hazard Management Using a Unified Software Environment Numerical Simulation Tool "RAMMS"," in Congress Interpraevent, 2012, pp. 77-86.

[7] R. I. Leine, A. Schweizer et al., "Simulation of rockfall trajectories with consideration of rock shape," Multibody Sys. Dyn., 2014.

[8] F. Bourrier, F. Berger et al., "Rockfall rebound: comparison of detailed field experiments and alternative modelling approaches," Earth Surface Processes and Landforms, vol. 37, no. 6, pp. 656-665, 2012.

[9] L. K. A. Dorren, "Rockyfor3d revealed description of the complete $3 d$ rockfall model,," Tech. rep., EcorisQ,, 2010.
[10] H. Lan, C. D. Martin, and C. Lim, "Rockfall analyst: A GIS extension for three-dimensional and spatially distributed rockfall hazard modeling," Computers \& Geosciences, vol. 33, no. 2, 2007.

[11] G. Ma, H. Matsuyama et al., "Practical studies on rockfall simulation by DDA," JRMGE, vol. 3, no. 1, pp. 57-63, 2011.

[12] H. Masuya, K. Amanuma et al., "Basic rockfall simulation with consideration of vegetation and application to protection measure," NHESS, vol. 9, no. 6, 2009

[13] F. Agliardi and G. Crosta, "High resolution three-dimensional numerical modelling of rockfalls," International Journal of Rock Mechanics and Mining Sciences, vol. 40, no. 4, pp. 455 - 471, 2003.

[14] C. L. Jones, J. D. Higgins, and R. D. Andrew, "Colorado Rockfall Simulation Program: Version 4.0," 2000.

[15] G. B. Crosta and F. Agliardi, "Parametric evaluation of 3D dispersion of rockfall trajectories," NHESS, vol. 4, no. 4, pp. 583-598, 2004.

[16] G. Chen, L. Zheng et al., "Numerical simulation in rockfall analysis: a close comparison of 2-d and 3-d dda," Rock mechanics and rock engineering, vol. 46, no. 3, pp. 527-541, 2013.

[17] A. Preh, A. Mitchell et al., "Stochastic analysis of rock fall dynamics on quarry slopes," International Journal of Rock Mechanics and Mining Sciences, vol. 80, pp. 57-66, 2015.

[18] S. Zihajehzadeh, T. J. Lee et al., "Integration of mems inertial and pressure sensors for vertical trajectory determination," IEEE TIM, vol. 64 , no. 3, pp. 804-814, 2015.

[19] D. A. Grejner-Brzezinska, C. K. Toth et al., "A robust solution to highaccuracy geolocation: Quadruple integration of gps, imu, pseudolite, and terrestrial laser scanning," IEEE TIM, vol. 60, no. 11, 2011.

[20] A. Bergeron and N. Baddour, "Design and development of a low-cost multisensor inertial data acquisition system for sailing," IEEE TIM, vol. 63, no. 2, pp. 441-449, 2014.

[21] S. Sabatelli, M. Galgani et al., "A double-stage kalman filter for orientation tracking with an integrated processor in 9-d imu," IEEE TIM, vol. 62, no. 3, pp. 590-598, 2013.

[22] P. Niklaus, T. Birchler et al., "StoneNode: A Low-Power Sensor Device for Induced Rockfall Experiments," in IEEE SAS, 2017.

[23] F. Bourrier, L. Dorren et al., "Toward objective rockfall trajectory simulation using a stochastic impact model," Geomorph., vol. 110, no. 3-4, pp. 68-79, 2009.

[24] W. D. Stevens, "RocFall, a tool for probabilistic analysis, design of remedial measures and prediction of rockfalls," University of Torronto, Tech. Rep., 1998.

[25] A. Volkwein, B. Fritschi et al., "Spatial Tracking of a falling Rock using internal Acceleration Sensors," SLF, Davos, Switzerland, Tech. Rep., 2005.

[26] A. Volkwein and J. Klette, "Semi-automatic determination of rockfall trajectories," Sensors, vol. 14, no. 10, 2014.

[27] S. Marchetty, "Analysis of Rockfall Trajectories and Evaluation of Concrete Barrier Efficiency," Akron Univ., Tech. Rep., 2015.

[28] F. Berger and L. Dorren, "Objective comparison of rockfall models using real size experimental data," Disaster mitigation of debris flows, slope failures and landslides, pp. 245-252, 2006.

[29] O. Gronz, P. H. Hiller et al., "Smartstones: A small 9-axis sensor implanted in stones to track their movements," CATENA, vol. 142, pp. 245 - 251, 2016.

[30] R. P. Narayanan, T. V. Sarath, and V. V. Vineeth, "Survey on motes used in wireless sensor networks: Performance \& parametric analysis," Wireless Sensor Network, vol. 8, no. 04, p. 67, 2016.

[31] "FatFs - Generic FAT File System Module," 2016, accessed Nov. 2016. [Online]. Available: http://elm-chan.org/fsw/ff/00index_e.html

[32] E. D. Sneed and R. L. Folk, "Pebbles in the Lower Colorado River, Texas a Study in Particle Morphogenesis," The Journal of Geology, vol. 66, no. 2, pp. 114-150, 1958.

[33] SD Group (Panasonic, SanDisk, Toshiba), "SD Specifications Part 1, Physical Layer - Simplified Specification," SD Card Association, Tech. Rep. 4.10, Jan. 2013.

[34] Y. Bühler, M. S. Adams et al., "Photogrammetric Reconstruction of Homogenous Snow Surfaces in Alpine Terrain Applying Near-Infrared UAS Imagery," IJRS, vol. 38, no. 8-10, pp. 3135-3158, 2017. 